\title{
Achieving sensitive, high-resolution laser spectroscopy at CRIS
}

\author{
R. P. de Groote ${ }^{1}$ K. M. Lynch ${ }^{2}$. \\ S. G. Wilkins ${ }^{3}$ the CRIS collaboration
}

Published online: 13 December 2016

(C) The Author(s) 2016. This article is published with open access at Springerlink.com

\begin{abstract}
The Collinear Resonance Ionization Spectroscopy (CRIS) experiment, located at the ISOLDE facility, has recently performed high-resolution laser spectroscopy, with linewidths down to $20 \mathrm{MHz}$. In this article, we present the modifications to the beam line and the newly-installed laser systems that have made sensitive, high-resolution measurements possible. Highlights of recent experimental campaigns are presented.
\end{abstract}

Keywords CRIS · ISOLDE · Laser spectroscopy · Resonance ionization

\section{Introduction}

The Collinear Resonance Ionization Spectroscopy (CRIS) experiment combines the two standard techniques of laser spectroscopy. The collinear geometry of fast-beam laser spectroscopy, allowing high resolution measurements, is combined with the high-detection efficiency of the resonance-ionization technique. With the CRIS experiment located at the radioactive beam facility ISOLDE, this provides the ability to perform highly-sensitive laser spectroscopy measurements on exotic isotopes.

This article is part of the Topical Collection on Proceedings of the 10th International Workshop on Application of Lasers and Storage Devices in Atomic Nuclei Research: "Recent Achievements and Future Prospects" (LASER 2016), Poznań, Poland, 16-19 May 2016

Edited by Krassimira Marinova, Magdalena Kowalska and Zdzislaw Błaszczak

K. M. Lynch

kara.marie.lynch@cern.ch

1 KU Leuven, Instituut voor Kern- en Stralingsfysica, 3001 Leuven, Belgium

2 ISOLDE, EP Department, CERN, CH-1211 Geneve 23, Switzerland

3 School of Physics and Astronomy, The University of Manchester, Manchester M13 9PL, UK 


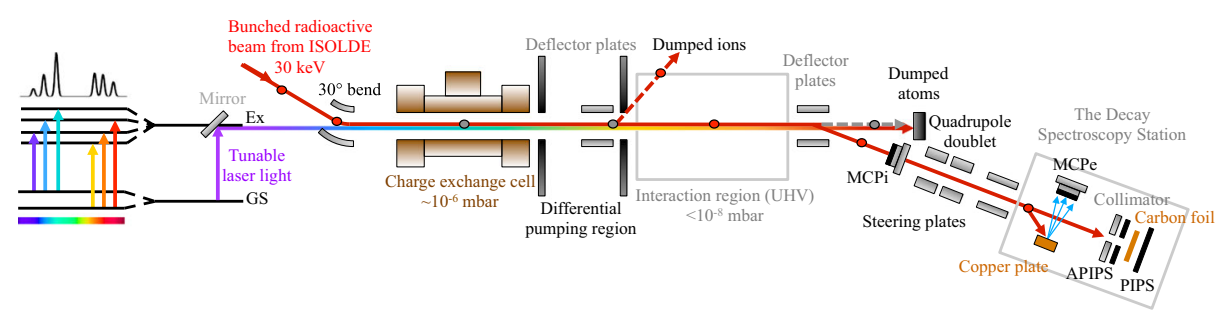

Fig. 1 [Color online] Schematic of the CRIS beam line. The tunable laser light is overlapped in a collinear geometry with the atomic bunch, allowing the hyperfine structure of the isotope under investigation to be probed. Step-wise excitation and ionization of the isotope produces ions that can be detected either with MCPi, MCPe or the DSS. See text for details

Laser spectroscopy, and the study of the hyperfine structure of isotopes, is a nuclearmodel independent method of measuring fundamental properties of the nucleus, such as nuclear spin. Measurement of the $A$ and $B$ hyperfine parameters allows for the extraction of the magnetic dipole moment and spectroscopic quadrupole moment, respectively. Additionally, the isotope shift from one isotope to another can provide insights into an isotope's change in mean-square charge radius. The ability to resolve the complete hyperfine structure, with as narrow linewidth as possible, allows precise measurements of these properties to be performed.

Since the initial low-resolution studies of the francium isotopes [1-3], the CRIS experiment has focused on performing high-resolution laser spectroscopic measurements, while improving the total experimental efficiency. This article presents the recent modifications to the CRIS setup that has resulted in high-resolution studies of exotic francium, gallium and copper isotopes.

\section{The CRIS experiment}

The heart of the CRIS setup [4, 5] is the interaction region, held at $<10^{-8}$ mbar, where the collinear resonance ionization process occurs, see Fig. 1. In order to prepare the atoms for resonant ionization, the bunched radioactive-ion beam produced by ISOLDE is first passed through a charge-exchange cell. Here, the ion bunch is neutralised via collisions with potassium vapour held at $\approx 450 \mathrm{~K}$. The non-neutralised ions are deflected by an electrostatic plate, and the neutral atoms pass into the $1.3 \mathrm{~m}$ interaction region. Here, the tunable laser light is overlapped with the atomic bunch in a collinear geometry, inducing resonant excitation of an electron from one fine-structure state to another, probing the isotope's hyperfine structure. Additional beams of laser light are overlapped, both spatially and temporally, with the resonant transition, at the frequency necessary to ionize the atom. Atoms not ionized (typically isobaric contaminants) travel to a beam dump. The ions produced from resonant ionization are detected with a positive-ion multichannel plate (MCPi) held at $-2.4 \mathrm{kV}$, or impinged on a copper dynode and the emitted secondary electrons detected with $\mathrm{MCPe}$, biased at $+2.4 \mathrm{kV}$. Alternatively, the ions can be deflected to the Decay Spectroscopy Station (DSS) for alpha-tagging of hyperfine components and laser-assisted nuclear decay spectroscopy, utilizing the silicon PIPS detectors $[6,7]$. 


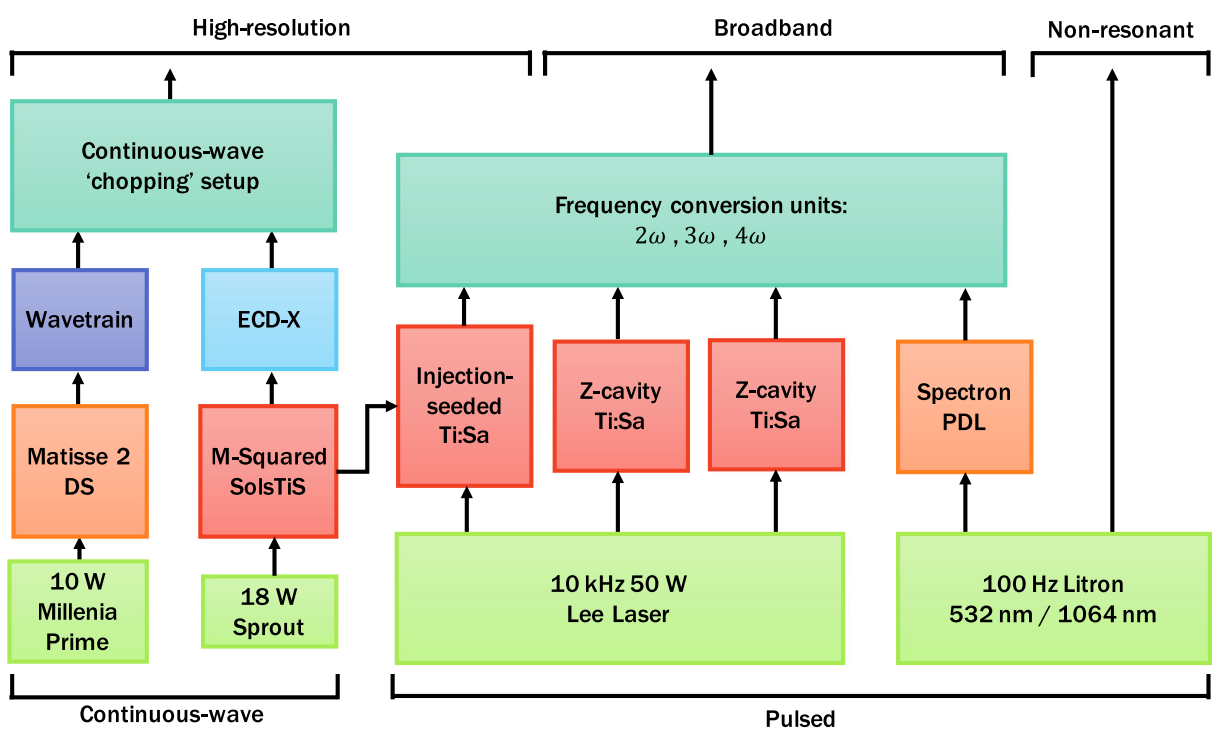

Fig. 2 Schematic diagram of the laser systems at CRIS. There are 3 high-resolution tunable laser systems available, in addition to 3 broad-band lasers

\section{Recent modifications}

The recent modifications made to the CRIS setup have aimed to benefit two of the main principles of the technique: reduction of the laser linewidth in order to perform high-resolution measurements, and enhancement of the experimental efficiency for low-yield isotope studies. Production of narrow-linewidth laser light has been achieved with installation of several new laser systems. Increasing the experimental efficiency took the form of improving the ion-beam transport and detection efficiency through installation of several beam monitoring and detection devices.

\subsection{Towards high-resolution laser spectroscopy}

Extending the CRIS technique to new cases has necessitated significant investment in additional laser systems to allow greater flexibility in developing ionization schemes. The majority of these are installed in a new laser laboratory adjacent to the ISOLDE facilty. Laser light produced in the CRIS laser laboratory can be either mirror- or fibre-coupled into the CRIS beam line. An overview of the lasers available at CRIS is shown in Fig. 2.

There are 3 high-resolution tunable laser systems available for measuring an isotope's hyperfine structure: an M-Squared SolsTiS continuous-wave titanium-sapphire laser, a Matisse 2 DS continuous-wave dye laser and an injection-seeded titanium-sapphire laser developed at the IGISOL facility at the University of Jyväskylä [8]. The M-Squared SolsTiS produces up to $6 \mathrm{~W}$ of infrared light that can be frequency-doubled by an ECD-X frequency-doubler to produce up to $2 \mathrm{~W}$ of blue light whilst light from the Matisse $2 \mathrm{DS}$ can be frequency-doubled by a Wavetrain 2 frequency-doubler.

To prevent optical pumping to dark states, the continuous-wave light from these lasers is 'chopped' into pulses. This is achieved by sending the light through a Pockels cell and 
(a)

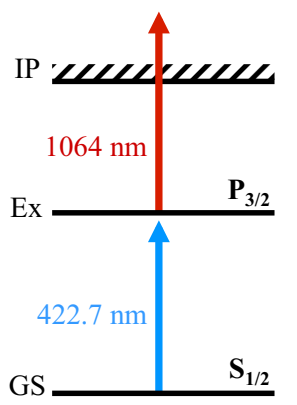

(b)
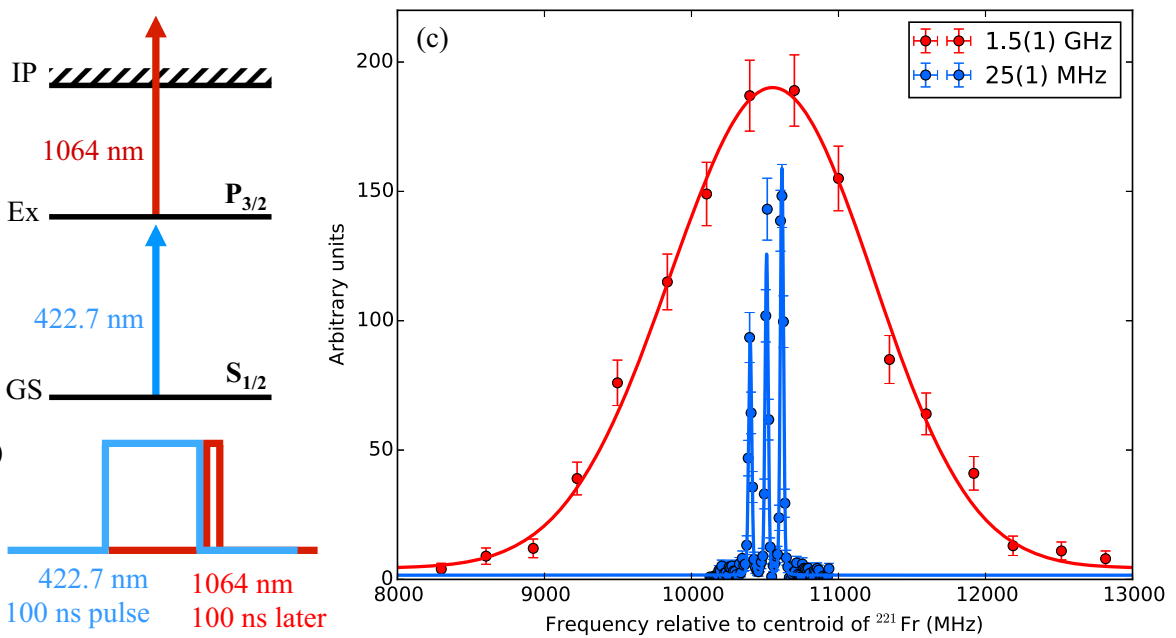

Fig. 3 From low-resolution to high-resolution collinear resonance ionization spectroscopy of ${ }^{221}$ Fr. a Twostep ionization scheme for francium, with the resonant transition from $7 s^{2} S_{1 / 2} \rightarrow 8 p^{2} P_{3 / 2}$ at $422.7 \mathrm{~nm}$. b Schematic of the cw-laser light 'chopping' method used to achieve high-resolution measurements. $\mathbf{c}$ Righthand component of the hyperfine structure of ${ }^{221} \mathrm{Fr}$. In red, initial low-resolution measurements with a linewidth of $1.5(1) \mathrm{GHz}$, which could only resolve the lower-state $7 s^{2} S_{1 / 2}$ splitting. In blue, 25(1) MHz linewidths were achieved, enough to resolve the upper-state $8 p^{2} P_{3 / 2}$ splitting

a polarization analyser. This analyser is tuned such that the intensity of the laser light is reduced by a factor of 2000 if no voltage is applied to the Pockels cell. However, if a high voltage is applied, typically $2.4 \mathrm{kV}$, the polarization of the light is rotated by 90 degrees, which maximizes the intensity of the laser light going to the CRIS beamline. Therefore, by applying short voltage pulses on the Pockels cell, short light pulses are created. More details on the method can be found in [9]. Schematic diagrams of the resonance-ionization scheme of francium and the cw-laser light 'chopping' method are shown in Fig. 3a and b, respectively. Figure $3 \mathrm{c}$ illustrates the improvement in resolution of the hyperfine structure of ${ }^{221} \mathrm{Fr}$ from $1.5(1) \mathrm{GHz}$ to 20 (1) $\mathrm{MHz}$ when this method was used. A key advantage of this approach is that the length and timing of the laser pulse can be easily and precisely controlled. For example, it is advantageous to adjust the length of the laser pulse based on the lifetime of the atomic excited state. This way, a balance can be made between maximizing the efficiency of the excitation process and minimizing optical pumping processes.

The M-Squared SolsTiS can also provide seed light for the injection-seeded system which allows high-resolution light to be produced. An advantage of such a system is that efficient single-pass higher harmonic generation with non-linear crystals becomes possible. By frequency tripling or quadrupling light from the injection-seeded system, deep-UV highresolution laser light can be produced with power densities sufficient for efficient excitation in a multi-step resonance ionization scheme. The injection locked laser system therefore provides additional wavelengths that a continuous wave laser system cannot produce. Both the injection locked laser system and the chopped cw laser system have demonstrated efficient excitation, even for weak transitions.

A new Lee Laser LDP-100MQG $10 \mathrm{kHz} \mathrm{Nd:YAG} \mathrm{laser} \mathrm{capable} \mathrm{of} \mathrm{producing} \mathrm{up} \mathrm{to}$ $50 \mathrm{~W}$ of $532 \mathrm{~nm}$ light has been installed to pump the injection-seeded system and two 


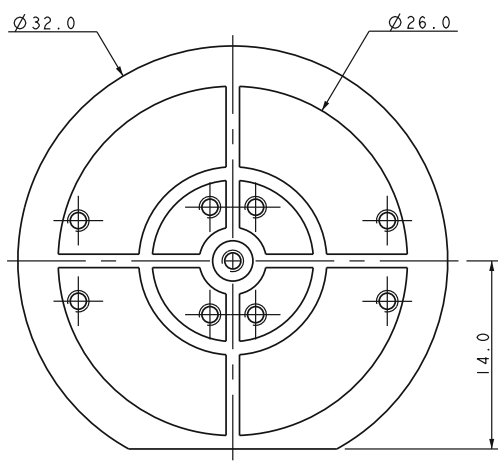

(a)

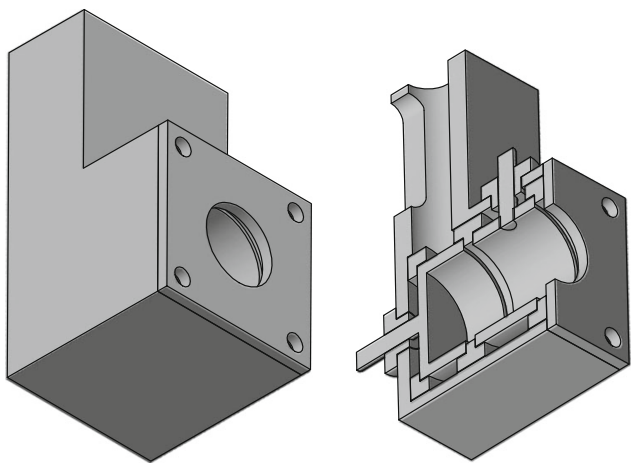

(b)

Fig. 4 a Technical drawing of the segmented Faraday cup, a printed circuit board in a radial design. The central segment has a diameter of $3 \mathrm{~mm}$, the middle segments have an outer diameter of $10 \mathrm{~mm}$, and the outer segments have a diameter of $26 \mathrm{~mm}$. Each segment is separated by $1 \mathrm{~mm}$. b 3D representations, complete and cutaway, of the suppressed Faraday cups

Z-cavity titanium-sapphire lasers on loan from the LARISSA group at Johannes GutenbergUniversität Mainz. Two new Z-cavity systems are currently being constructed that will be permanently installed at CRIS. A Spectron pulsed dye laser pumped by a Litron LPY 601 50-100 PIV Nd:YAG laser is also available. The Z-cavity systems and pulsed dye laser produce broadband light to further excite to a higher-lying state or auto-ionizing state. The Litron has two independent laser heads each capable of producing $80 \mathrm{~mJ}$ of $1064 \mathrm{~nm}$ light or $50 \mathrm{~mJ}$ of $532 \mathrm{~nm}$ light at $100 \mathrm{~Hz}$ repetition rate. If a suitable auto-ionizing state doesn't exist, $1064 \mathrm{~nm}$ or $532 \mathrm{~nm}$ light from the Litron can be use to complete the ionization process non-resonantly.

The wavelength of the laser systems can be measured by either a HighFinesse WSU2 or WS6-600 wavelength meter, which provides an active feedback to the scanning software of each laser system, allowing the lasers to be scanned and locked. The WSU2 wavelength meter is calibrated by a temperature-stabilized helium neon laser.

\subsection{Enhancing the experimental efficiency}

Initially, the CRIS setup was installed with a negative-ion MCP (MCPe, Hamamatsu F465512), see Fig. 1, to detect the secondary emission of electrons when the resonantly-ionized ions were impinged onto a copper dynode. Located in the DSS, $1.8 \mathrm{~m}$ away from the deflector plates, this MCP suffers from ion-beam transmission losses. In order to address this issue, an additional positive-ion MCP (MCPi, Hamamatsu F4655-12) was installed, $53 \mathrm{~cm}$ downstream of the deflector plates. Initial tests have measured a transmission efficiency of $40 \%$ together with a detection efficiency of $80 \%$. This compares favourably to the nominal combined detection and transmission efficiency of $\approx 30 \%$ for MCPe.

In order to optimise the overlap of the atomic bunch with the laser beams, a segmented Faraday cup was installed at the end of the interaction region. This 'Faraday cup', in actuality a printed circuit board, has a radial design with 9 segments, see Fig. 4a. The central copper segment has a diameter of $3 \mathrm{~mm}$, the middle 4 segments have an outer diameter of $10 \mathrm{~mm}$, and the outer 4 segments a diameter of $26 \mathrm{~mm}$. Each segment is individually electrically connected (via Kapton-coated copper cables) to a 9-pin UHV type-C sub-miniature 
electrical feed-through to maintain the high-vacuum conditions required. During beamtuning, the current induced by the ion beam on the central segment is optimized, while the individually-measured currents on the other segments are simultaneously minimized. This ensures the ion (atom) beam is travelling through the central axis of the interaction region, and the spatial overlap with the laser beams is maximal.

In addition, two unsuppressed Faraday cups in the CRIS beam line were exchanged for suppressed models, see Fig. 4b. These, located after the charge exchange cell and the interaction region (before the segmented Faraday cup), provide better accuracy for determining beam-transport efficiency. All installed beam-monitoring devices are mounted on remotecontrolled pneumatic linear drives (Kurt J. Lesker KLPDBAP) to ensure reproducible positioning along the beamline axis.

Stable-beam tuning through the CRIS setup has been improved by installation of multiple ETPS common-ground high voltage supplies (modules HTP-EHS 80-60x-105F and HTPEHS 40-100x-504F installed in a HTP-EHS 40-100x-504F mainframe). With 44 channels available (36 channels of $\pm 6 \mathrm{kV}$ and 8 channels of $\pm 10 \mathrm{kV}$ ), each is remotely-controllable via a Python-controlled CAN interface. An automatic beam-tuning programme has recently been implemented in the control of the high-voltage power supplies, allowing optimization of the magnitude of the incident ion beam on a Faraday cup or MCP. The programme is based on the robust conjugate direction search method developed by Huang et al. [10]. It is derivative free and therefore robust to the noise on the signal, making it ideal for online optimization.

\section{Recent high-resolution CRIS results}

The recent modifications to the CRIS beam line and laser laboratory have yielded sensitive, high-resolution measurements of eoxtic francium, gallium and copper isotopes.

The investigation into the evolution of nuclear structure in neutron-deficient francium was furthered by measuring ${ }^{203} \mathrm{Fr}$ in high-resolution [11]. $100 \mathrm{~mW}$ of $422.7 \mathrm{~nm}$ laser light was produced from frequency-doubled light from the M-Squared SolsTiS, allowing the hyperfine structure of the $7 s^{2} S_{1 / 2} \rightarrow 8 p^{2} P_{3 / 2}$ transition to be studied with a linewdith of $20 \mathrm{MHz}$. The measured quadrupole moment from this experiment compliments previous low-resolution studies of the isotope at CRIS [1], allowing a more complete picture of the nuclear wave function to be obtained.

High-resolution hyperfine-structure studies of both neutron-rich (around $N=50$ ) and neutron-deficient $(A=65,67)$ gallium isotopes were performed, marking the first three-step resonance ionization scheme at CRIS [12]. The first resonant step probed the $4 p^{2} P_{3 / 2} \rightarrow 5 s^{2} S_{1 / 2}$ transition at $417.2 \mathrm{~nm}$, with a $40 \mathrm{MHz}$ linewidth. The laser light was produced by the M-Squared SolsTiS and ECD-X laser system, with a peak power of $150 \mathrm{~mW}$ entering the CRIS interaction region.

The magicity of ${ }^{78} \mathrm{Ni}$ can be studied through laser spectroscopy of the neutron-rich copper isotopes. High-resolution CRIS measurements provided magnetic dipole moments, electric quadrupole moments, spins and charge radii for ${ }^{76-78} \mathrm{Cu}$ [13]. These isotopes have been previously unstudied in high-resolution due to their low production yields of down to $20 / \mathrm{s}$ for ${ }^{78} \mathrm{Cu}$. The copper atoms were laser ionized with a resonant $249 \mathrm{~nm}$ $4 s^{2} S_{1 / 2} \rightarrow 4 p^{4} P_{3 / 2}$ excitation step and a second step to an auto-ionizing state using $314 \mathrm{~nm}$. The $0.2 \mu \mathrm{J} /$ pulse UV light for the excitation step was produced with the injectionseeded system, locked to light from the M-Squared system, which proved stable throughout the experiment. Analysis of all three high-resolution data sets is currently ongoing. 


\section{Summary}

High-resolution collinear resonance ionization spectroscopy has recently been performed with the CRIS experiment at ISOLDE. Through installation of several narrow-linewidth laser systems in the new CRIS laser laboratory, alongside beam-monitoring and detection devices in the CRIS beam line, high-resolution hyperfine-structure measurements have been performed on exotic isotopes of francium, gallium and copper. With a laser suite capable of providing a variety of high-resolution and broadband laser frequencies for resonance ionization schemes, the CRIS setup is ready for forthcoming experimental campaigns of low-yield exotic isotopes.

Acknowledgments We acknowledge the support of the ISOLDE collaboration and technical teams. This work was supported by ERC Consolidator Grant No.648381; the Science and Technology Facilities Council Consolidated Grant No.ST/F012071/1, Continuation Grant No.ST/J000159/1 and Ernest Rutherford Grant No.ST/L002868/1; the IUAP-Belgian State Belgian Science Policy (BRIX network P7/12), FWOVlaanderen (Belgium) and GOA 10/010 from KU Leuven; and the European Union's Seventh Framework Programme for Research and Technological Development under Grant Agreements: 262010 (ENSAR), 267194 (COFUND), and 289191 (LA3NET). K. M. L. was supported by the FWO Pegasus Marie Curie Fellowship under Grant No. 267216. T.E.C. was supported by the STFC Ernest Rutherford Fellowship No. ST/J004189/1. K. T. F. was supported by STFC Advanced Fellowship Scheme Grant No. ST/G006415/1.

Open Access This article is distributed under the terms of the Creative Commons Attribution 4.0 International License (http://creativecommons.org/licenses/by/4.0/), which permits unrestricted use, distribution, and reproduction in any medium, provided you give appropriate credit to the original author(s) and the source, provide a link to the Creative Commons license, and indicate if changes were made.

\section{References}

1. Flanagan, K.T., Lynch, K.M., Billowes, J., et al.: Collinear resonance ionization spectroscopy of neutrondeficient francium isotopes. Phys. Rev. Lett. 111, 212501 (2013)

2. Lynch, K.M., Billowes, J., Bissell, M.L., et al.: Decay-assisted laser spectroscopy of neutron-deficient francium. Phys. Rev. X 4, 011055 (2014)

3. Budinčević, I., Billowes, J., Bissell, M.L., et al.: Laser spectroscopy of francium isotopes at the borders of the region of reflection asymmetry. Phys. Rev. C 90, 014317 (2014)

4. Procter, T.J., Aghaei-Khozani, H., Billowes, J., et al.: Development of the CRIS (Collinear Resonant Ionisation Spectroscopy) beam line. J. Phys. Conf. Series 318, 012070 (2012)

5. Cocolios, T.E., de Groote, R.P., Billowes, J., et al.: High-resolution laser spectroscopy with the Collinear Resonance Ionisation Spectroscopy (CRIS) experiment at CERN-ISOLDE. Nucl. Instrum. Meth. B 376, 284-287 (2016)

6. Lynch, K.M., Cocolios, T.E., Rajabali, M.M.: Laser assisted decay spectroscopy at the CRIS beam line at ISOLDE. Hyperfine Interact 216, 95-101 (2013)

7. Lynch, K.M., Cocolios, T.E., Billowes, J., et al.: Combined high-resolution laser spectroscopy and nuclear decay spectroscopy for the study of the low-lying states in ${ }^{206} \mathrm{Fr},{ }^{202} \mathrm{At}$, and ${ }^{198} \mathrm{Bi}$. Phys. Rev. C 93, 014319 (2016)

8. Sonnenschein, V.: Ph.D. Thesis, Department of Physics, University of Jyväskylä (2014)

9. de Groote, R.P., Budinčević, I., Billowes, J., et al.: Use of a continuous wave laser and Pockels cell for sensitive high-resolution collinear resonance ionization spectroscopy. Phys. Rev. Lett. 115, 132501 (2015)

10. Huang, X., Corbett, J., Safranek, J., Wu, J.: An algorithm for online optimization of accelerators. Nucl. Instrum. Meth. A 726, 77-83 (2013)

11. Wilkins, S.G. et al.: In preparation (2016)

12. Farooq-Smith, G.J. et al.: In preparation (2016)

13. de Groote, R.P. et al.: In preparation (2016) 